\title{
Dermatologie und gap junctions im Zeitalter der Molekulargenetik und Zellbiologie - tiefe Einblicke erwünscht
}

\section{Dermatology and Gap Junctions in the Age of Molecular Genetics and Cell Biology - Deep Insights Needed}

Autoren

Institute
M. A. M. van Steensel", J. Frank

Department of Dermatology und Maastricht University Center for Molecular Dermatology (MUCMD), University Hospital Maastricht, Maastricht, The Netherlands

\section{Bibliografie}

Dol 10.1055/s-2006-944859

Akt Dermatol 2006; 32;

457-462 @ Georg Thieme

Verlag KG Stuttgart · New York ISSN 0340-2541

Korrespondenzadresse

Dr. Maurice A. M. van Steensel

M. D., Ph. D.

Department of Dermatology University Hospital Maastricht P. Debyelaan 25; PO Box 5800 6202 AZ Maastricht

The Netherlands

mvst@sder.azm.nl

\section{Zusammenfassung \\ $\nabla$}

Es ist unbestreitbar, dass sich in den zurückliegenden Jahren ein steter Wandel in der Dermatologie vollzogen hat. Dies impliziert jedoch nicht allein, dass es sich bei unserem Fach schon lange nicht mehr um eine rein beschauende und beschreibende Disziplin handelt. Vielmehr sind wir derzeit auch therapeutisch und wissenschaftlich gesehen Zeuge einer sich leise und unaufhaltsam ausbreitenden Revolution, die gerade erst begonnen hat und deren Ende noch lange nicht abzusehen ist. Großen Anteil an den teilweise bahnbrechenden jüngsten Entwicklungen,

\section{Einleitung}

\section{$\nabla$}

„We wish to suggest a structure fort the salt of deoxyribose nucleic acid (D.N.A.). This structure has novel features which are of considerable biological interest.“ und „It has not escaped our notice that the specific pairing we have postulated immediately suggests a possible copying mechanism for the genetic material.“ - Diese berühmten Worte aus der bahnbrechenden Publikation über die Doppelhelix-Struktur der DNA im April 1953 in der Zeitschrift Nature [1] können unzweifelhaft zu den größten Untertreibungen in der Geschichte der genetischen Forschung und der Wissenschaft im allgemeinen gerechnet werden; insbesondere der zweite von uns zitierte Satz ist, im Nachhinein betrachtet, „understatement“ pur. Mit dieser Veröffentlichung läuteten Watson und Crick eine neue Epoche ein: das Zeitalter der Molekulargenetik. Nur etwas mehr als 50 Jahre später befinden wir uns bereits in einem neuen Jahrhundert, dem 21., um genau zu sein. Und eben dieses 21. Jahrhundert wird bereits jetzt von vielen denen mitunter sogar jahrzehntelange Dogmata in der Dermatologie zum Opfer fallen, haben die enormen Fortschritte auf dem Gebiet der Molekulargenetik und Zellbiologie. Auch unser Labor hat sich der modernen dermatologischen Forschung auf Basis molekulargenetischer und zellbiologischer Untersuchungstechniken verschrieben. In diesem Übersichtsartikel stellen wir am Beispiel von Syndromen, die durch Gap-junctionDefekte hervorgerufen werden, ausgesuchte Aspekte unserer umfangreichen wissenschaftlichen Tätigkeit auf dem Gebiet hereditärer Hauterkrankungen vor. als das „Zeitalter der Biologie“ betrachtet [2], ein Begriff, den man sicherlich breit auslegen und in verschiedenster Art und Weise interpretieren und analysieren kann.

Aus molekulargenetischer, entwicklungs- und zellbiologischer Sicht jedoch verleiht dieses Credo unterschwellig der Hoffnung Ausdruck, dass sich unser wissenschaftliches Verständnis der komplexen biologischen Vorgänge in Zellen und Organismen exponentiell derartig vergrößern möge, dass wir eventuell den Punkt erreichen, an dem wir unsereins nach Belieben und wie selbstverständlich manipulieren können - in etwa vergleichbar der Fähigkeit, Materie zu verändern. Von einer derartigen Entwicklung würden sicherlich alle biologisch-wissenschftlichen Disziplinen profitieren, auch und insbesondere die Medizin. Wir glauben, dass diese Entwicklung bereits ihren Anfang genommen hat, und möchten nachfolgend anhand einiger Beispiele darstellen, welchen Einfluss der gegenwärtig rasche wissenschaftliche Erkenntniszuwachs auf die Natur der dermatologischen Forschung hat.

* Preisträger der Berliner Stiftung für Dermatologie 


\section{Dermatologie im Wandel \\ $\nabla$}

Schon von altersher ist das Studium der Hauterkrankungen Individuen mit der Gabe der exakten Beobachtungsfähigkeit und genauen Detailanalyse vorbehalten gewesen. Diesen Persönlichkeiten haben wir die Effloreszenzenlehre zu verdanken sowie eine ausgeklügelte und detaillierte Nosologie, wie sie wahrscheinlich in keiner anderen medizinischen Disziplin zu finden ist. Dennoch war, betrachtet man die Geschichte der Dermatologie, ihr eigentlicher Gegenstand - die Haut - Jahrhunderte lang mehr oder weniger eine „Black Box“, also ein Organ, dessen innerer Aufbau und genaue innere Funktionsweise weitestgehend unverstanden geblieben ist. Während Dermatologen in der Lage waren eine minutiöse Beschreibung dessen zu geben, was sie sahen, und seit dem Einzug der topischen Glukokortikoide und Immunmodulatoren auch imstande sind, diese Hautveränderungen zu behandeln, so hatten und haben sie doch größtenteils Mühe zu erklären, warum und auf welche Weise nun eine bestimmte Therapie wirkt. So wissen wir noch stets nicht, wie Dithranol genau wirkt, um nur ein Beispiel zu nennen. Und dies, obwohl seit 1916 bekannt ist, dass es ausgezeichnet bei der Psoriasis wirkt und offensichtlich in der erkrankten Haut die Zellteilung und Keratinozytenproliferation hemmt, ohne dabei einen wesentlichen Einfluss auf die nicht von der Erkrankung betroffenen Hautareale auszuüben.

Seit kurzem jedoch beginnt sich die Situation zu wandeln, und ohne zu übertreiben darf man sagen, dass sich leise eine kleine Revolution anbahnt. So verfügen wir nunmehr beispielsweise über eine neue Generation von Medikamenten in der Behandlung der Psoriasis und anderer Erkrankungen, den so genannten Biologics, die auch als Biologicals oder Biologika bekannt sind. Biologics sind bio- bzw. gentechnologisch hergestellte Proteine, die körpereigenen Substanzen sehr ähnlich und spezifisch gegen ausgewählte Zelloberflächenmoleküle und -rezeptoren gerichtet sind, wie z.B. der TNF-alpha-Blocker Etanercept. Biologics greifen in verschiedene Regulationsmechanismen des Körpers ein und modulieren dabei unter anderem die Immunantwort. Somit können Biologics wesentlich spezifischer zur Beeinflussung einzelner Regulationsmechanismen eingesetzt werden und üben eine gezieltere Wirkung auf Krankheitsprozesse aus als andere allgemein und eher unspezifisch wirkende Medikamente. Neben den Biologics befinden sich auch bestimmte modifizierte Designerdrogen bereits in der klinischen Erprobung.

Durch den kontinuierlichen Fortschritt auf dem Gebiet der Molekulargenetik wird die genetische Basis einer stetig wachsenden Anzahl hereditärer Erkrankungen aufgedeckt, und immer öfters entpuppen sich als komplexe oder polygene Dermatosen angesehene Erkrankungen, wie z.B. die atopische Dermatitis, als Krankheitsbilder, die letztendlich auf einen spezifischen und eher simplen (Gen-)Defekt zurückgeführt werden können [3]. Daneben finden auch immer mehr Gentherapie- und Enzymsubstitutionsschemata sowie die RNA-Interferenz (RNAi) ihren Weg aus den Forschungslaboratorien in den klinischen Alltag [4]. Unser Labor im Zentrum für Molekulare Dermatologie der Universität Maastricht (Maastricht University Center for Molecular Dermatology; MUCMD) hat es sich zur Aufgabe gemacht, durch molekulargenetische und zellbiologische Untersuchungen seinen Anteil zur zuvor erwähnten Revolution beizusteuern. Als Beispiel für moderne dermatologische Forschung, wie sie von uns verstanden wird, stellen wir nachfolgend ausgesuchte Aspekte unserer wissenschaftlichen Tätigkeit auf dem Gebiet hereditärer Erkrankungen dar, die durch Defekte der so genannten gap junctions hervorgerufen werden.

\section{Kommunikation in der Dermatologie - gap junctions}

Die Haut ist ein äußerst kommunikationsfreudiges Organ. Die oberste Schicht der Haut, die Epidermis, ist überwiegend aus Keratinozyten aufgebaut, welche einem permanenten Proliferations- und Differenzierungsprozess unterworfen sind und eine hohe Dichte an so genannten gap junctions aufweisen. Bei gap junctions handelt es sich um ubiquitär im Körper vorkommende interzelluläre Kanäle, die aus Connexinen bestehen. Connexine sind transmembranöse Strukturproteine der Zell-Zell-Kanäle ( $\triangle$ Abb. 1) und vermitteln die rasche Kommunikation zwischen epidermalen Zellen.

Connexine gehören zu einer großen Proteinfamilie, die im gesamten Tierreich verbreitet ist. Sie können zum einen auf Basis ihres jeweiligen Molekulargewichtes klassifiziert (z. B. Connexin 26), zum anderen aber auch in die Subfamilien A, B und C unterteilt werden, wobei sich die letztgenannte Klassifikation an gleichartigen Sequenzen auf Nukleotid- und Proteinebene orientiert. Gegenwärtig werden beide Nomenklaturen der Einfachheit halber variabel gebraucht. Connexin-Proteine setzen sich aus vier Transmembrandomänen zusammen. Hierbei sind das Nund C-terminale Ende intrazellulär gelegen, so dass sich als Folge zwei extrazelluläre Schleifen bilden. Jeweils sechs ConnexinMoleküle formen in der Zellmembran ein Connexon, das auch als Hemikanal bezeichnet wird. Jeweils zwei Connexon-Hexamere benachbarter Zellen verbinden sich miteinander und formen hierdurch direkte interzytoplasmatische gap junction-Kanäle. Diese Kanäle kann man sich am besten als einen Schlauch mit wässrigem Inhalt vorstellen, der die Passage von Wasser und kleinen Botenmolekülen erlaubt. Die Permeabilität dieser Kanäle wird durch verschiedene Faktoren reguliert, darunter insbesondere die Membranpolarisierung. Durch das Öffnen der Connexon-Kanäle wird die Kommunikation und Signalinteraktion zwischen benachbarten Zellen ermöglicht, vornehmlich über elektrische Kopplungsmechanismen und den Austausch kleiner Moleküle. Da sich Connexone aus mehreren verschiedenen Connexinen zusammensetzen können, weist die hieraus resultierende Kanalstruktur mitunter eine sehr komplexe Komposition auf, welche die jeweils spezifischen funktionellen Eigenschaften dieser Kanäle determiniert - ein Prozess, den wir in diesem Augenblick nur rudimentär verstehen [5].

Über die gap junctions in ihren Zellmembranen kommunizieren Keratinozyten mit Melanozyten, dermalen Blutgefäßen und verschiedenen Zellen des Immunsystems, die in die Epidermis eindringen können [6]. Daher ist es wahrscheinlich auch nicht verwunderlich, dass strukturelle Defekte in Connexinen zu schweren hereditären Hauterkrankungen führen können [5] und pathologische Veränderungen in der gap junction-Kommunikation mit hyperproliferativen Dermatosen wie Psoriasis und Plattenepithelkarzinomen assoziiert sind [7]. Aus therapeutischen Überlegungen heraus wäre es somit wünschenswert, die Gapjunction-Aktivität zielgerichtet modulieren zu können [8]. Um dieses Ziel zu erreichen, müssten wir jedoch erst verstehen, was die genaue Funktion der gap junctions in der Haut ist. Derzeit bestehen lediglich recht allgemeine Hinweise darauf, dass gap junctions in der Epidermis die delikate Balance zwischen Proliferation und Differenzierung regulieren. Obwohl wir also im Grunde nicht sehr viel über die pathophysiologische Bedeutung und exakte Funktion der gap junctions in der Haut wissen, hat sich dennoch unser Verständnis von den funktionellen Aufgaben dieser Proteine durch das Studium hereditärer Erkrankungen, 

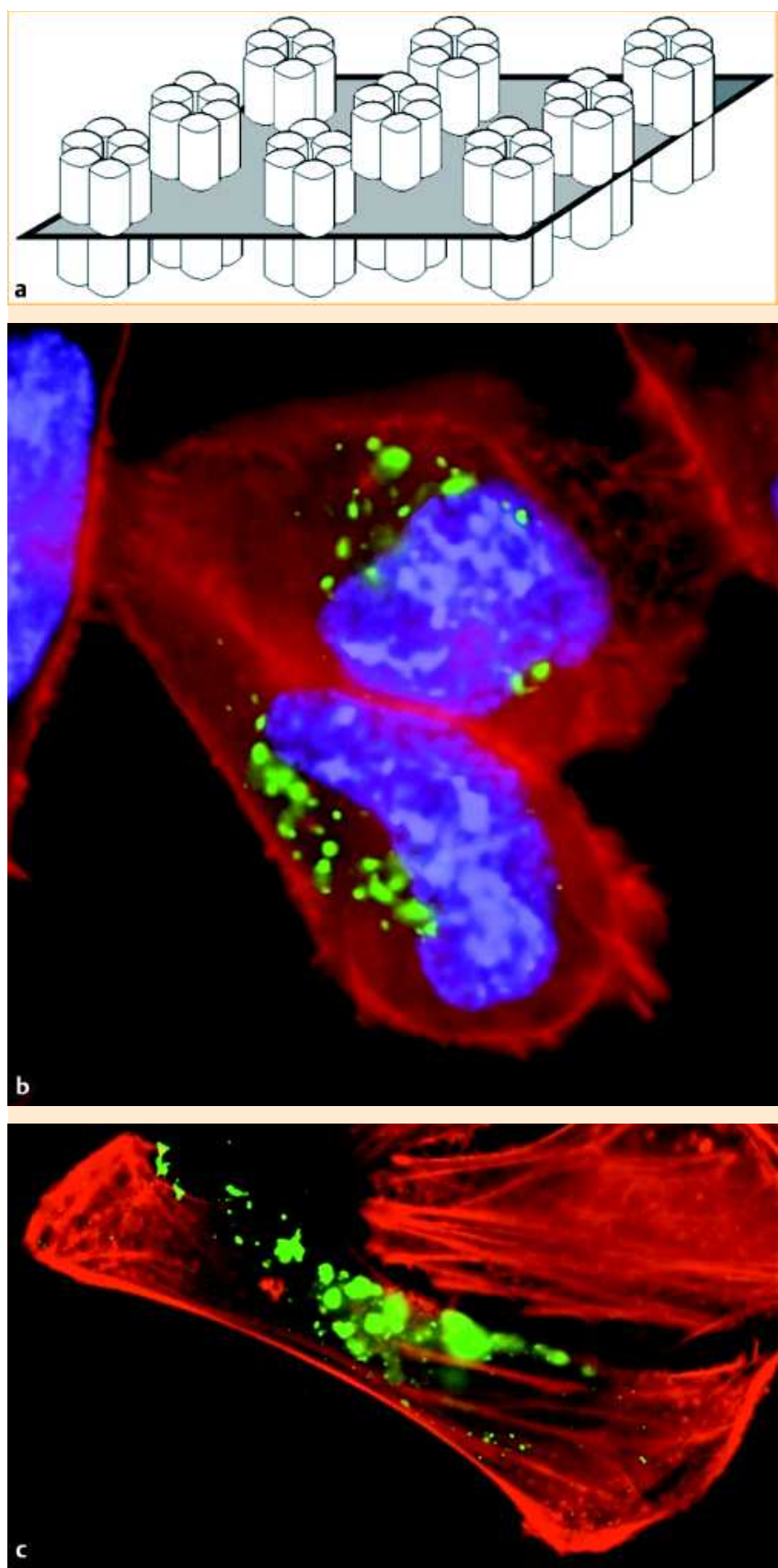

Abb. 1 a Gap-junction-Kanäle in einer Zellmembran. b GJB2-D50N Mutiertes Protein (grün) in HeLa Zellen. c GJB2-D66H.

die durch Mutationen in für gap junctions kodierende Genen hervorgerufen werden, in den zurückliegenden Jahren ständig vergrößert.

Derzeit sind mindestens 14 verschiedene Gene bekannt, die für Transmembranproteine aus der Connexin-Familie kodieren [8]. Eines der interessantesten kutan exprimierten Gene aus dieser Familie ist GJB2. Dieses Gen kodiert für das Protein Connexin 26 (Cx 26), eigentlich ein relativ unbedeutsames Mitglied der Connexin-Familie in der Haut, das jedoch ein ganz spezielles Charakteristikum aufweist: aus pathogenen DNA-Sequenzabweichungen (Mutationen) im GJB2-Gen resultierende Aminosäurensubstitutionen an spezifischen, aber unterschiedlichen Positionen des Proteins resultieren phänotypisch unterschiedliche Erkrankungen mit einem zuweilen spektakulären Facettenreichtum an klinischen Symptomen.
So führt z.B. die Punktmutation G12R, bei der es zur Aminosäurensubstitution von Glycin durch Arginin kommt, zum so genannten Keratitis-Ichthyosis-Deafness (KID)-Syndrom (OMIM 148210) [9], das durch sensorische Taubheit und eine schwere Keratodermie gekennzeichnet ist. Patienten mit KID-Syndrom weisen darüber hinaus eine erhöhte Infektneigung auf, erkranken vermehrt an Mykosen und entwickeln häufiger Plattenepithelkarzinome. Neben der zuvor genannten Mutation G12R führen auch die Punktmutationen S17F (Substitution von Serin durch Phenylalanin) und D50N (Substitution von Asparaginsäure durch Asparagin) zum KID-Syndrom [10]. Dies allein ist sicher nicht weiter verwunderlich, wäre da nicht die Tatsache, dass zahlreiche andere Aminosäurensubstitutionen in Cx 26 zu völlig verschiedenartigen Krankheitsbildern führen. N14K (Substitution von Asparagin durch Lysin) resultiert im Hypotrichosis-Deafness-Syndrom (OMIM 129500), das neben dünnen Haaren und Taubheit durch Nageldystrophie und eine mild ausgeprägte Erythrokeratodermie gekennzeichnet ist [11]. Interessanterweise ist der mutierte Asparagin-Rest an Position 14 des Cx 26 in unmittelbarer Nachbarschaft zweier Aminosäuren, G12 und S17, gelegen, die beim KID-Syndrom mutiert sind. Bewegen wir uns weiter in 3'-Richtung auf das carboxyterminale Ende des Cx 26 zu, so fällt auf, dass die Punktmutation G59A (Substitution von Glycin durch Alanin) mit einem Krankheitsbild assoziiert ist, das sich klinisch mit Taubheit und einer palmoplantaren Keratodermatose manifestiert (palmoplantar keratoderma-deafness syndrome; OMIM 121011) [12]. Demgegenüber führt die Punktmutation D66H (Substitution von Asparaginsäure durch Histidin) zum Vohwinkel-Syndrom, das durch eine teilweise mutilierende palmoplantare Keratodermatose und Innenohrschwerhörigkeit charakterisiert ist [13]. Nur einem Ignoranten könnte entgehen, dass die Substitution dreier Aminosäurenreste in Cx 26, die in enger räumlicher Beziehung zueinander gelegen sind, D50, G59 und D66, zu völlig verschiedenen Erkrankungen führen. Doch damit nicht genug: die Liste der Mutationen in Cx 26 ist lang und wird ständig länger, ohne dass ein Ende in Sicht wäre ( $\bullet$ Tab. 1). Warum nun Mutationen in Cx 26 zu phänotypisch derart unterschiedlichen Krankheitsbildern führen ist eine der Fragen, deren Beantwortung sich derzeit neben unserer eigenen Arbeitsgruppe sicherlich auch andere Forschungsgruppen weltweit intensiv widmen. Die einzige Konstante im Spektrum der zuvor erwähnten Mutationen ist ihre klinische Assoziation mit Taubheit bzw. Schwerhörigkeit, da Cx 26 erforderlich ist, um das Transmembranpotential der Haarzellen im Innenohr aufrecht zu erhalten [14].

Betrachtet man die verschiedenen Mutationen so gewinnt man das Gefühl, dass die Rolle des Cx 26 in der Haut etwas komplexer ist. So könnten beispielsweise einzelne Aminosäuren innerhalb des Proteins spezifische Funktionen wahrnehmen. Plausibler jedoch wäre die alternative Erklärung, dass Cx 26 aus einer Reihe funktioneller Module besteht, die durch die diversen Mutationen differentiell in ihrer Funktion beeinträchtigt werden und in der Folge zu gleichartigen aber doch verschiedenen Hauterkrankungen führen. Die jüngsten Publikationen diverser Arbeitsgruppen und unserer eigenen deuten darauf hin, dass letztere Hypothese der Wirklichkeit am nächsten kommen dürfte. Gapjunction-Proteine weisen die nützliche Eigenschaft auf, dass ihre Funktionalität nicht eingeschränkt wird, wenn man sie experimentell mit einer fluoreszierenden Signalsequenz versieht. Hierdurch kann man den Weg der Botenmoleküle durch die Zelle visualisieren und die Auswirkungen analysieren, die einzelne Mutationen auf den Transport des Proteins vom Endoplasmati- 
Tab. 1 Genotyp-Phänotyp Korrelation für GJB2

\begin{tabular}{|c|c|}
\hline Erkrankung & Mutation in GJB2 \\
\hline Hypotrichosis-Taubheit & N14K \\
\hline Keratitis-Ichthyosis-Taubheit & G12R, S17F, G45E, D50N, D50Y \\
\hline KID-ähnlich & $\mathrm{N} 14 \mathrm{Y}$ \\
\hline Bart-Pumphrey-Syndrom & N54K \\
\hline PPK-Taubheit & G59A, G59S, R75Q, R75W \\
\hline Vohwinkel Syndrom & $\mathrm{D} 66 \mathrm{H}$ \\
\hline Mucositis-Taubheit-Syndrom & F142L \\
\hline
\end{tabular}

schen Retikulum, seinem Entstehungsort, zur Zellmembran haben. Mit Hilfe dieser Technologie können wir nun demonstrieren, dass einige der diversen phänotypischen Charakteristiken der Mutationen im GJB2-Gen durch ihre Effekte auf den intrazellulären Proteintransport erklärt werden können. So konnten wir beispielsweise zeigen, dass die mit dem KID-Syndrom assoziierte Punktmutation D50N keinerlei Einfluss auf den Proteintransport hat, sondern das mutierte Protein direkt in die Zellmembran integriert wird ( $\bullet$ Abb. 1 b). Andere Arbeitsgruppen konnten demonstrieren, dass es dort als konstitutionell aktiver Kanal wirkt und seine Signalfunktion ohne externen Stimulus ausübt (Gonzalez et al.; persönliche Mitteilung). Daraus geht hervor, dass es sich bei D50N um eine Mutation mit Aktivitätsgewinn (so genannte „gain of function“-Mutation) handelt. Demgegenüber resultiert die Mutation D66H in einem Transportdefekt, der dafür verantwortlich ist, dass das Protein im Golgi- oder Post-Golgi-Netzwerk verbleibt ( $\bullet$ Abb. 1 c). Daher wird das mutierte Protein auch wahrscheinlich nicht in der Zellmembran exprimiert und verändert hierdurch entscheidend die Komposition der verbleibenden Gap-junction-Kanäle. Unsere jüngsten experimentellen Befunde deuten darauf hin, dass sich beim Vohwinkel-Syndrom und auch bei vergleichbaren klinischen Phänotypen möglicherweise eine Genotyp-Phänotyp-Korrelation abzeichnet und Syndrome, die mit Taubheit und palmoplantarer Keratodermie assoziiert sind, generell durch Mutationen hervorgerufen werden, die einen Proteintransportdefekt zur Folge haben (de Zwart et al.; Manuskript in Vorbereitung).

Selbstverständlich sind die Vorgänge in der Natur niemals simpel, und so können auch die zuvor beschriebenen Befunde nicht erklären, warum Veränderungen in einem eher unbedeutenden Gap-junction-Protein zu derartig schweren hereditären Hauterkrankungen führen können. Ein weiterer Effekt, den Mutationen im GJB2-Gen ausüben können, ist der verminderte Einbau anderer Connexine in die Zellmembran [15]. Ein derartiger Effekt, den man als transdominant bezeichnet, konnte bereits für $\mathrm{Cx} 30$ (GJB6) und Cx 43 (GJA1) aufgezeigt werden, zwei Proteine aus der Connexin-Familie, die prominent in der Haut exprimiert sind.

Insbesondere GJA1 ist von vorrangigem Interesse für uns, da es das bedeutendste Gap-junction-Protein in der Haut ist. Wenn die Expression von GJA1 unter dem direkten Einfluss von GJB2 stünde würde dies eine gute Erklärung dafür liefern, warum sich als Folge verschiedener GJB2-Mutationen hauptsächlich Hautsymptome manifestieren, obwohl dieses Gen für ein in der Haut relativ unbedeutsames Gap-junction-Protein kodiert. Erstaunlicherweise sind jedoch die durch Mutationen im GJA1-Gen hervorgerufenen Erkrankungen üblicherweise nicht mit kutanen Symptomen assoziiert.
Das Mysterium um Connexin 43 - was macht es in der Haut?

$\nabla$

Mutationen im GJA1-Gen verursachen die oculo-dento-digitale Dysplasie (ODDD) (OMIM 164200). Hierbei handelt es sich um ein komplexes Syndrom, dass sich mit ophthalmologischen Abweichungen, Hypertelorismus, Hypoplasie der ala nasi und akral betonten Skelettdefekten manifestiert, darunter vornehmlich eine Typ III-Syndaktylie und einer Hypoplasie der mittleren Phalanx der digiti V [16]. Kürzlich wurden auch Defekte in der substantia alba des zentralen Nervensystems beschrieben, die neurologische Symptome wie spastische Paraplegie und Urininkontinenz hervorrufen können [17]. Dies ist nicht erstaunlich, da Cx 43 eines der wichtigsten Gap-junction-Proteine in Astrozyten ist. In den Astrozyten geht es eine enge Bindung mit Cx 46.6 (GJA12) ein. Mutationen im für Connexin 46.6 kodierenden GJA12-Gen verursachen ein autosomal rezessiv vererbtes, dem Pelizaeus-Merzbacher-Syndrom ähnliches Krankheitsbild (OMIM 608804), das durch Defekte in der Substantia alba gekennzeichnet und unter anderem mit Nystagmus, progressiver Spastizität und Ataxie assoziiert ist [18].

Es ist jedoch weitestgehend unklar, wie diese Symptome entstehen, obwohl es Hinweise darauf gibt, dass Cx 43 von essentieller Bedeutung für die Sonic Hedgehog-Signalkaskade und die Entwicklung spezifischer Gesichtsstrukturen ist $[19,20]$. Noch erstaunlicher ist in diesem Zusammenhang, dass Hautsymptome bei der ODDD fehlen. So dachte man jedenfalls bis vor kurzem... Kürzlich jedoch haben wir mehrere Patienten mit ODDD und einer variabel ausgeprägten palmoplantaren Keratodermie beschrieben [21]. Diese unerwarteten Befunde beantworten dann auch die Frage, was Cx 43 in der Haut macht - denn wenn Mutationen im GJA1-Gen nicht zu kutanen Abweichungen führen, läge die Vermutung nahe, dass es für die Haut überflüssig ist. Unsere Ergebnisse belegen das Gegenteil. Und mehr noch: Sie unterstützen eindrucksvoll die Hypothese, dass Mutationen in Cx 26 auch Auswirkungen auf Cx 43 haben. Konkret konnten wir aufzeigen, dass unsere Patienten einen neuen Mutationstyp aufwiesen. Alle bis dahin beschriebenen Nukleotidabweichungen bei ODDD waren Punkmutationen wie z. B. G130R (Substitution von Glycin durch Arginin) [22]. Im Gegensatz dazu fanden wir bei unseren Patienten Dinukleotid-Deletionen, die das carboxyterminal lokalisierte zytoplasmatische Endstück des Cx 43 beeinflussen. Diese Deletion von zwei Basenpaaren führt zu einer Veränderung des Leserahmens (rahmenverschiebende Mutation), zum Einbau falscher Aminosäuren und zu einem vorzeitigen Kettenabbruch im kodierten Protein auf Grund eines präterminalen Stop-Kodons. Als Konsequenz beinhaltet das Endstück des kodierten $\mathrm{Cx}$-43-Proteins, verglichen mit dem Wildtyp-Protein, eine unsinnige Aminosäurensequenz und ist zu kurz. Wir konnten derartige Mutationen bei mehreren, nicht miteinander verwandten Patienten mit ODDD und palmoplantarer Keratodermie nachweisen, was dafür spricht, dass Hautveränderungen spezifisch mit Dinukleotid-Deletionen assoziiert sind, die im carboxyterminalen Endstück des Cx 43 auftreten. Da dieser Mutationstyp mit einem charakteristischen klinischen Phänotyp assoziiert ist, haben wir die Hypothese entwickelt, dass carboxyterminale Deletionen, im Vergleich zu Punktmutationen im GJA1-Gen, auch spezifische funktionelle Konsequenzen für das CX-43-Protein haben könnten. Jüngste Ergebnisse aus unserer Gruppe (de Zwart et al.; unpublizierte Daten und Vreeburg M et al., in press) und von anderen [23] bestätigen diese Vermutung: während rahmenverschiebende Mutationen wie 
die Dinukleotid-Deletionen einen dominant-negativen Transportdefekt verursachen, wird ein solcher bei Punktmutationen nicht beobachtet. 0 Abb. 2 zeigt die Hautbiopsie eines unserer Patienten mit der Mutation C260fsX307. Man erkennt deutlich, dass sowohl das Wildtyp- als auch das mutierte Protein im Zytoplasma zur Expression kommen und sich dort anhäufen. Im Gegensatz dazu wird ein Fusionsprotein, das die Punktmutation G130R beinhaltet, in die Zellmembran transportiert ( $\bullet$ Abb. 2). Auch ein um das carboxyterminale Endstück trunkiertes $\mathrm{Cx}$ 43-Protein wird normal in die Zellmembran transportiert [23]. Daher vermuten wir, dass es durch den bei unseren Patienten beobachteten Einbau einer falschen und unsinnigen Aminosäurensequenz in das carboxyterminale Endstück des Proteins zu einer Transportstörung kommt, möglicherweise durch eine Interferenz mit der physiologischen Gap-junction-Komposition. Unabhängig vom präzisen Pathomechanismus weisen unsere Untersuchungsbefunde darauf hin, dass die Hautsymptome bei diesen Patienten durch die Abwesenheit von $\mathrm{Cx} 43$ in der Zellmembran verursacht werden. Diese Erkenntnisse sind in Übereinstimmung mit dem zuvor beschriebenen Befund, dass mit Hautveränderungen assoziierte Mutationen in GJB2 in einer verminderten Expression von Cx 43 im Gap-junction-Plaque resultieren.

\section{Schlussfolgerungen und Zukunftsausblick}

$\nabla$

In dieser Übersicht haben wir einige unserer jüngsten Forschungsergebnisse vorgestellt und diskutiert, um zu zeigen, wie Fragen und Probleme in der Dermatologie durch den Einsatz moderner molekulargenetischer und zellbiologischer Untersuchungstechniken angegangen und aufgelöst werden können. Zur Veranschaulichung unserer Untersuchungsstrategien haben wir die Gap-junction-Erkrankungen als Bespiel gewählt, weil sie zum einen im Fokus unserer derzeitigen Forschungsbemühungen stehen, zum anderen aber auch, weil diese Proteinfamilie, wie zuvor beschrieben, durch ganz spezielle Eigenschaften und Charakteristika in der menschlichen Haut gekennzeichnet ist und unsere Befunde verdeutlichen, wie die Lösung eines Problems dazu beitragen kann, ein weiteres ebenfalls aufzulösen oder sich daraus ableitende Fragen zu beantworten.

Die gegenwärtige dermatologische Forschung nutzt modernste wissenschaftliche Untersuchungstechniken und entwickelt sich sehr schnell - die brandaktuellen Forschungsergebnisse von heute sind die Lehrbucheinträge von morgen. Unser sich stetig vergrößerndes Verständnis basaler pathophysiologischer $\mathrm{Zu}$ sammenhänge wird eher früher als später dazu beitragen, dass heute im Labor gewonnene Erkenntnisse möglichst bald schon im klinischen Alltag zur Erweiterung derzeitiger Therapiestrategien und zur Verbesserung der Patientenversorgung beitragen. Aber auch in umgekehrter Richtung können wir eine Menge lernen. Da wir heutzutage ein wesentlich kompletteres Bild über die genetische Vielfalt zahlreicher Hauterkrankungen haben und stets besser verstehen, in welcher Assoziation bestimmte Mutationen zu spezifischen Hautabweichungen stehen, gelingt es uns auch immer häufiger, neue nosologische Kriterien voneinander zu unterscheiden, derer wir uns früher nicht bewusst waren. Als ein Beispiel sei hier die lamelläre Ichthyose genannt, die bis vor kurzem einfach nur als solche bekannt war. Heutzutage unterscheiden wir jedoch sechs verschiedene Typen der lamellären Ichthyose [24], und es hat sich herausgestellt, dass jede dieser Varianten ihre eigenen, sie von den anderen Formen un-
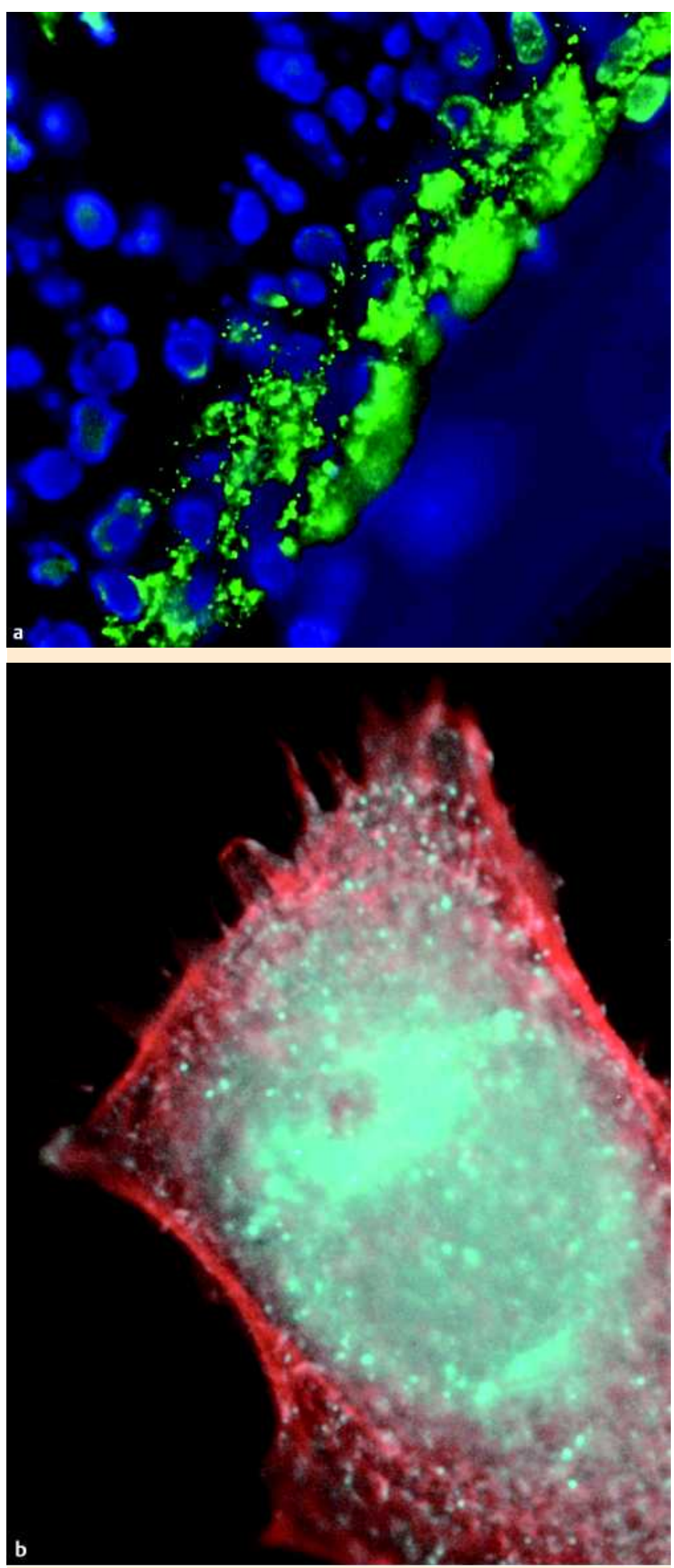

Abb. 2 a GJA1-C260fsX307. Mutiertes und normales Protein im Zytoplasma bei einer ODDD-Patientin. b GJA1-G130R. Das mutierte Protein ist blau.

terscheidenden Merkmale hat - wenn man erst einmal weiß, wonach man schauen muss. Wir sind daher fest davon überzeugt, dass wir aufregenden und spannenden Zeiten entgegensehen können, die innovative Therapiekonzepte und eine noch ausgeklügeltere Nosologie mit sich bringen werden. Dies wird dazu führen, dass die Dermatologie endgültig die ihr mancherorts noch stets anhaftende Aura des rein beschauenden und de- 
skriptiven Faches abstreifen kann, um wie Phönix der Asche zu entsteigen und schließlich den ihr gebührenden Platz im Mittelpunkt der modernen biologisch-naturwissenschaftlichen Disziplinen einzunehmen.

\section{Danksagung|Forschungsförderung $\nabla$}

Maurice A. M. van Steensel wird unterstützt durch Stipendien der Berliner Stiftung für Dermatologie und des Forschungsinstituts GROW im Universitätsklinikum Maastricht, den Europäischen Forschungspreis 2005 der Firma La Roche Posay und Barrier Therapeutics NV. Jorge Frank ist Mitglied des Netzwerks für Ichthyosen und verwandte Keratinisierungsstören (NIRK), das durch das Bundesministerium für Bildung und Forschung (BMBF) unterstützt wird.

\section{Abstract}

\section{Dermatology and Gap Junctions in the Age of Molecu- lar Genetics and Cell Biology - Deep Insights Needed $\nabla$}

Dermatology has slowly but surely been changing during the past few years. We are witnessing a silent revolution that is turning our discipline from a purely descriptive one into an interventional one that has at its disposal an ever increading arsenal of designer drugs. These developments are largely due to the recent and dramatic progress in molecular genetics and cell biology. Thanks to our increased insight into the molecular biology of the skin, age-old dogmata are overturned by new scientific insights and novel treatments for previously untreatable disorders are on the horizon. Our laboratory, too, has joined the revolution. In this review, we discuss our work on gap junctions to illustrate how modern molecular genetics and cell biology are contributing to our understanding of skin diseases.

\section{Literatur}

1 Watson JD, Crick FH. Molecular structure of nucleic acids; a structure for deoxyribose nucleic acid. Nature 1953; 171: 737-738

2 Satava RM. Biointelligence age: implications for the future of medicine. Stud Health Technol Inform 2001; 81: vii-X

3 Palmer CN, Irvine AD, Terron-Kwiatkowski A, Zhao Y, Liao H, Lee SP, Goudie DR, Sandilands A, Campbell LE, Smith FJ, O'Regan GM, Watson RM, Cecil JE, Bale SJ, Compton JG, DiGiovanna JJ, Fleckman P, Lewis-Jones $S$, Arseculeratne G, Sergeant A, Munro CS, El Houate B, McElreavey K, Halkjaer LB, Bisgaard H, Mukhopadhyay S, McLean WH. Common lossof-function variants of the epidermal barrier protein filaggrin are a major predisposing factor for atopic dermatitis. Nat Genet 2006; 38: $441-446$

4 Grunweller A, Hartmann RK. RNA interference as a gene-specific approach for molecular medicine. Curr Med Chem 2005; 12: 3143 - 3161

5 van Steensel MA. Gap junction diseases of the skin. Am J Med Genet 2004; 131C : $12-19$

6 Simon AM, McWhorter AR. Role of connexin 37 and connexin 40 in vascular development. Cell Commun Adhes 2003; 10: 379-385
7 Lucke T, Choudhry R, Thom R, Selmer IS, Burden AD, Hodgins MB. Upregulation of connexin 26 is a feature of keratinocyte differentiation in hyperproliferative epidermis, vaginal epithelium, and buccal epithelium. J Invest Dermatol 1999; 112: 354-361

8 Vinken M, Vanhaecke T, Papeleu P, Snykers S, Henkens T, Rogiers V. Connexins and their channels in cell growth and cell death. Cell Signal 2006; $18: 592-600$

9 Richard G, Rouan F, Willoughby CE, Brown N, Chung P, Ryynanen M, Jabs EW, Bale SJ, DiGiovanna JJ, Uitto J, Russell L. Missense Mutations in GJB2 Encoding Connexin-26 Cause the Ectodermal Dysplasia Keratitis-Ichthyosis-Deafness Syndrome. Am J Hum Genet 2002; 70: 5

10 van Steensel MA, van Geel M, Nahuys M, Smitt JH, Steijlen PM. A novel connexin 26 mutation in a patient diagnosed with keratitis-ichthyosis-deafness syndrome. J Invest Dermatol 2002; 118: 724 - 727

11 Van Steensel MA, Steijlen PM, Bladergroen RS, Hoefsloot EH, Van Ravenswaaij-Arts CM, Van Geel M. A Phenotype Resembling the Clouston Syndrome with Deafness Is Associated with a Novel Missense GJB2 Mutation. J Invest Dermatol 2004; 123: 291 - 293

12 Heathcote K, Syrris P, Carter ND, Patton MA. A connexin 26 mutation causes a syndrome of sensorineural hearing loss and palmoplantar hyperkeratosis (MIM 148350). J Med Genet 2000; 37: 50-51

13 Maestrini E, Korge BP, Ocana-Sierra J, Calzolari E, Cambiaghi S, Scudder $P M$, Hovnanian A, Monaco AP, Munro CS. A missense mutation in connexin26, D66H, causes mutilating keratoderma with sensorineural deafness (Vohwinkel's syndrome) in three unrelated families. Hum Mol Genet 1999; 8: 1237-1243

14 Kikuchi T, Adams JC, Miyabe Y, So E, Kobayashi T. Potassium ion recycling pathway via gap junction systems in the mammalian cochlea and its interruption in hereditary nonsyndromic deafness. Med Electron Microsc 2000; 33: 51 - 56

15 Rouan F, White TW, Brown N, Taylor AM, Lucke TW, Paul DL, Munro CS, Uitto J, Hodgins $M B$, Richard G. Trans-dominant inhibition of connexin-43 by mutant connexin-26: implications for dominant connexin disorders affecting epidermal differentiation. J Cell Sci 2001; 114 (Pt 11): $2105-2113$

16 Gillespie FD. A hereditary syndrome: „dysplasia oculodentodigitalis“. Arch Ophthal 1964; 71: 187-192

17 Loddenkemper T, Grote K, Evers S, Oelerich M, Stogbauer F. Neurological manifestations of the oculodentodigital dysplasia syndrome. J Neurol 2002; 249: 584-595

18 Uhlenberg B, Schuelke M, Ruschendorf F, Ruf N, Kaindl AM, Henneke M, Thiele H, Stoltenburg-Didinger G, Aksu F, Topaloglu H, Nurnberg P, Hubner C, Weschke B, Gartner J. Mutations in the gene encoding gap junction protein alpha 12 (connexin 46.6) cause Pelizaeus-Merzbacher-like disease. Am J Hum Genet 2004; 75: 251 - 260

19 Law LY, Lin JS, Becker DL, Green CR. Knockdown of connexin43-mediated regulation of the zone of polarizing activity in the developing chick limb leads to digit truncation. Dev Growth Differ 2002; 44: 537-547

20 McGonnell IM, Green CR, Tickle C, Becker DL. Connexin43 gap junction protein plays an essential role in morphogenesis of the embryonic chick face. Dev Dyn 2001; 222: 420-438

21 van Steensel MA, Spruijt L, van der Burgt I, Bladergroen RS, Vermeer M, Steijlen PM, van Geel M. A 2-bp deletion in the GJA1 gene is associated with oculo-dento-digital dysplasia with palmoplantar keratoderma. Am J Med Genet A 2005; 132A: 171 - 174

22 Paznekas WA, Boyadjiev SA, Shapiro RE, Daniels O, Wollnik B, Keegan $C E$, Innis JW, Dinulos MB, Christian C, Hannibal MC, Jabs EW. Connexin 43 (GJA1) mutations cause the pleiotropic phenotype of oculodentodigital dysplasia. Am J Hum Genet 2003; 72: 408-418

23 Maass K, Ghanem A, Kim JS, Saathoff M, Urschel S, Kirfel G, Grummer R, Kretz M, Lewalter T, Tiemann K, Winterhager E, Herzog V, Willecke K. Defective epidermal barrier in neonatal mice lacking the C-terminal region of connexin43. Mol Biol Cell 2004; 15: 4597-608

24 Oji V, Traupe H. Ichthyoses: differential diagnosis and molecular genetics. Eur J Dermatol 2006; 16: 349-359 